\title{
Congenital Cardiac Defects, Such as Eustachian Valve, May Increase the Risk of Cryptogenic Stroke: A Case Report
}

\author{
Chiara Bandinelli', Giovanni Maria Puddu'1, Anna Balducci², Giampaolo Bianchi' ${ }^{1}$ Marco Zoli ${ }^{1}$ \\ ${ }^{1}$ U.O. Stroke Unit, Policlinico di Sant'Orsola, Bologna, Italy \\ ${ }^{2}$ U.O. Cardiologia Pediatrica e dell'Età Evolutiva, Policlinico di Sant'Orsola, Bologna, Italy \\ Email: chiara.bandinelli2@studio.unibo.it
}

How to cite this paper: Bandinelli, C., Puddu, G.M., Balducci, A., Bianchi, G. and Zoli, M. (2020) Congenital Cardiac Defects, Such as Eustachian Valve, May Increase the Risk of Cryptogenic Stroke: A Case Report. World Journal of Cardiovascular Diseases, 10, 379-384.

https://doi.org/10.4236/wjcd.2020.106036

Received: February 26, 2020

Accepted: June 25, 2020

Published: June 28, 2020

Copyright $\odot 2020$ by author(s) and Scientific Research Publishing Inc. This work is licensed under the Creative Commons Attribution International License (CC BY 4.0).

http://creativecommons.org/licenses/by/4.0/

\begin{abstract}
Eustachian valve is an embryonic endocardial heart structure; after birth it becomes an embryogenic residue and its persistance in adult life is unusual. The literature reports some cases of association between Eustachian valve and PFO and is known that the prominent Eustachian valve can represent a way facilitating systemic embolism. Several studies also investigated the role of Eustachian valve in the pathophysiology of both migraine and cerebral embolism. In addiction is known how Eustachian valve may increase the risk of endocarditis, which mostly affects intravenous drug abusers or those with implanted medical devices or central venous catheters. The most commonly identified organism is Staphylococcus aureus (approximately 53\% of cases). We present a case of a 46-year-old woman with known migraine. She was hospitalized in Stroke Unit for a cryptogenic stroke with a right-to-left shunt detected with transcranial doppler ultrasound with "bubble test" and a patent foramen ovale with right-to-left shunt with a fenestration of atrial septum and a voluminous Eustachian valve detected with transesophageal echocardiography; she developed fever with a blood cultures positive for methicillin-resistant Staphylococcus aureus (MRSA), even if without evidence of endocarditis vegetations. In summary, the objective of our paper is to present an example of a correlation between the persistence of the Eustachian valve with both cryptogenetic stroke, possible valve infection and migraine.
\end{abstract}

\section{Keywords}

Eustachian Valve, Cryptogenic Stroke, Patent Foramen Ovale, PFO,

Endocarditis, Staphylococcus aureus, Transesophageal Echocardiography,

Migraine 


\section{Introduction}

Approximately one-half of patients with cryptogenic stroke have a patent foramen ovale (PFO).

A patent foramen ovale (PFO) is frequent and found in nearly $25 \%$ of healthy people. The majority of patients with PFO remain asymptomatic. The presence of $\mathrm{PFO}$, however, is higher in patients with cryptogenic stroke, suggesting there may be a group of patients with PFO who are certainly at risk for stroke because of the embolization through the PFO, which then pass to the cerebral circulation [1].

Transesophageal echocardiography (TEE) with bubble study is the noninvasive standard for diagnosing a PFO, permitting documentation of anatomic PFO features and atrial septal defect [2].

In embryonic heart, there is a valve, called Eustachian valve. It is an endocardial structure extending from the anterior margin of the inferior vena cava to the anterior part of the limbus fossa ovalis. It helps blood circulating through patent foramen ovale into the left atrium. After birth, Eustachian valve becomes an embryogenic residue. The persistence of Eustachian valve after birth is unusual. Transthoracic, transesophageal and three-dimensional echocardiography defines correctly diagnosis and anatomy of prominent Eustachian valve [3].

The literature reports some cases of association of Eustachian valve with PFO and is known that the prominent Eustachian valve can represent a way facilitating systemic embolism [3].

Several studies have investigated Eustachian valve in relation to cerebral embolism: a study conducted by Thomas A. Vale, James D. Newton et al. [4] analyzed some criteria to define which patients with PFO have more risk of developing stroke. The analysis was conducted on 72 patients, some parameters were measured related to the right atrium, such as the length of the VE, which may increase the risk of cerebral embolism.

Another study investigated the role of EV in the pathophysiology of both migraine and cerebral embolism. They enrolled 50 patients (mean age $37 \pm 12.5$ years) with previous stroke and migraine referred (diagnosed according to the International Headache Society criteria and Migraine Disability Assessment Score (MIDAS). After intracardiac echocardiography a prominent EV was diagnosticated in most patients with migraine. Patients with EV had more often a larger right-to-left shunt, more recurrent cerebral embolism before closure, and a higher preoperative MIDAS score. Patients with EV had a larger decrease in MIDAS score after closure [5].

In addiction is known how VE may increase the risk of endocarditis, which mostly affects intravenous drug abusers or those with implanted medical devices or central venous catheters. The most commonly identified organism is Staphylococcus aureus (approximately 53\% of cases). Treatment includes intravenous antibiotics for at least 4 weeks [6].

The objective of our paper is to show an example of correlation between spe- 
cific anatomical features, such as the persistence of Eustachian valve, and both cryptogenic stroke, endocarditis and migraine.

\section{Case Description}

A 46-year-old woman with known migraine presented to Emergency Department 2 hours after onset of headache, aphasia and motor right upper limb weakness.

The objective neurological examination signalled aphasia $3+2+2$, LOC commands 2 , best gaze 1 , visual fields 1 , facial paresia 1 , motor arm right 4 , motor leg right 3 (National Institute of Health Stroke Scale (NIHSS 19).

Computed tomography (CT) showed hyperdensity of left internal carotid artery; CT perfusion showed hypoperfusion in left middle cerebral artery territory with preserved penumbra volume (core 5\%); the CT angiography revealed occlusion of the left internal carotid and middle cerebral artery (MCA). No evidence of acute changes or hemorrhage.

She successfully received intravenous recombinant tissue-type plasminogen activator thrombolysis and thromebctomy within 2 hours and 40 minutes from stroke onset (LOC questions 2, facial paresis 1, aphasia 2, visual fields 1, motor arm right 2: NIHSS 8). Computed tomography (CT) showed left striatum subacute ischemic stroke, including left corona radiata and posterior capsule, with a small hemorrhage hyperdensity.

Transthoracic echocardiography didn't show any abnormalities. Transcranial doppler ultrasound with "bubble test" detected a right-to-left shunt. The ROPE score (Risk Of Paradoxical Embolism) was 8.

Transesophageal echocardiography revealed a patent foramen ovale with rightto-left shunt with a fenestration of atrial septum $(3 \mathrm{~mm}$ ) (Figure 1(a)) with a right-to-left shunt and a voluminous Eustachian valve, adherent to septum, with compartment of right atrium (Figure 1(b), Figure 1(c)).

Then the patient developed fever (TC $\max 38^{\circ} \mathrm{C}$ ). The white blood cell count was $10.73 \times 10^{9} / \mathrm{L}$, procalcitonin $<0.1 \mathrm{ng} / \mathrm{mL}$. Inflammatory markers were elevated, with a C-reactive protein of $4.95 \mathrm{mg} / \mathrm{L}$. Blood cultures were positive for methicillin-resistant Staphylococcus aureus (MRSA) and the urine cultures were positive for Escherichia coli and Enterococcus faecalis. The patient was underwent central venous catheter implantation; she denied any intravenous drug use. The transesophageal echocardiography had not seen endocarditis signs, however she was started on daptomycin plus piperacillin-tazobactam, which was later de-escalated to oxacillin once sensitivity of Enterococcus faecalis was confirmed, for 4 weeks. Follow up blood cultures after beginning antibiotic therapy was negative.

Surgery will be performed within one year with a complete closure of atrial septal defect with Gore Septal Occluder [7] by transfemoral approach, meanwhile she will continue antiaggregant therapy until the surgery.

After closure the patient will receive an instrumental and clinic follow up with transcranial doppler ultrasound (6 months and 12 months after surgery) and 


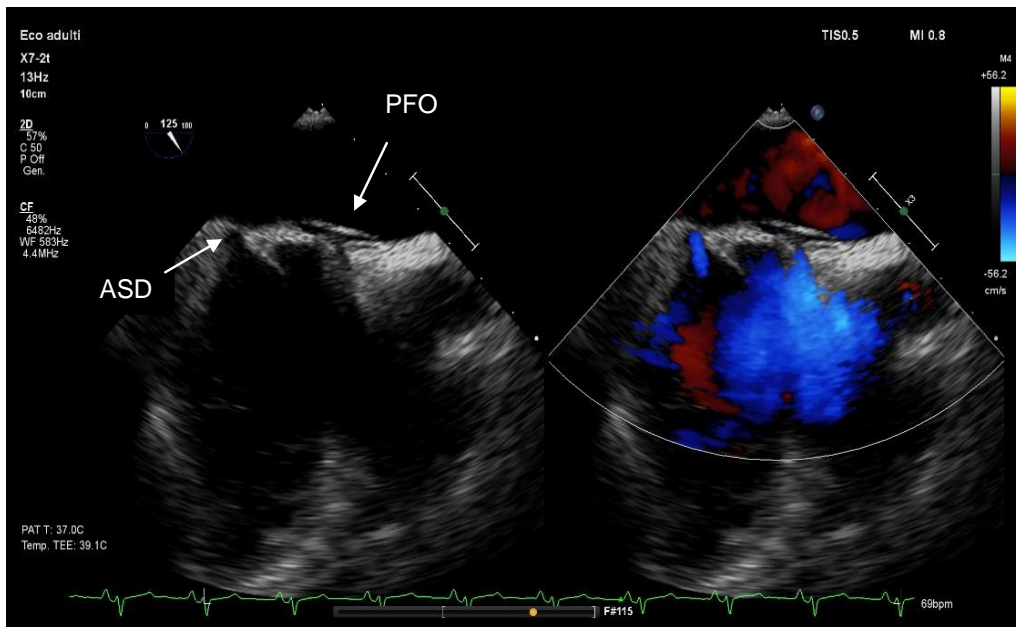

(a)

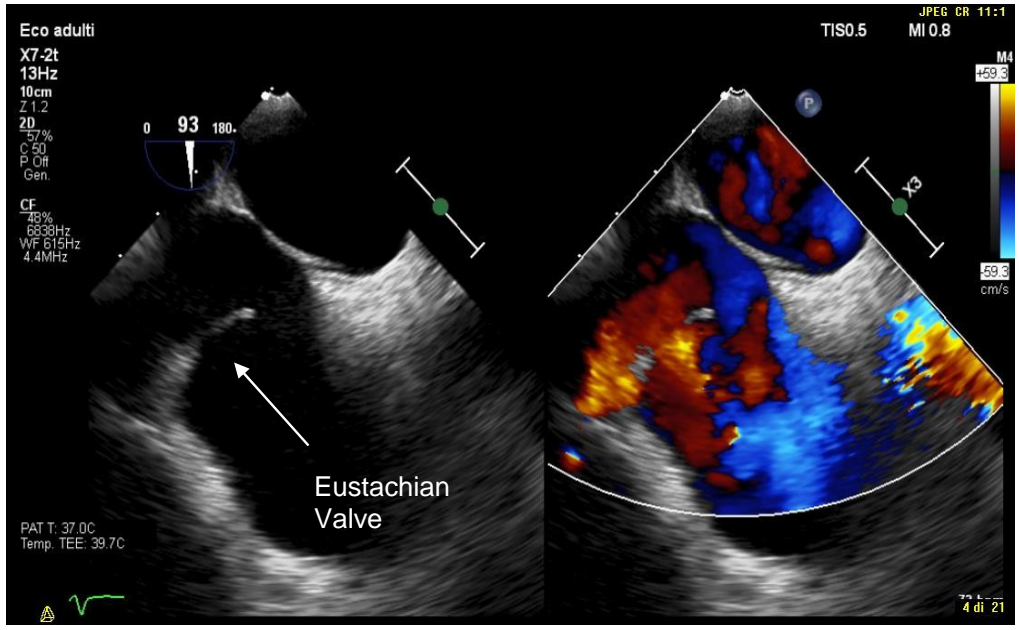

(b)

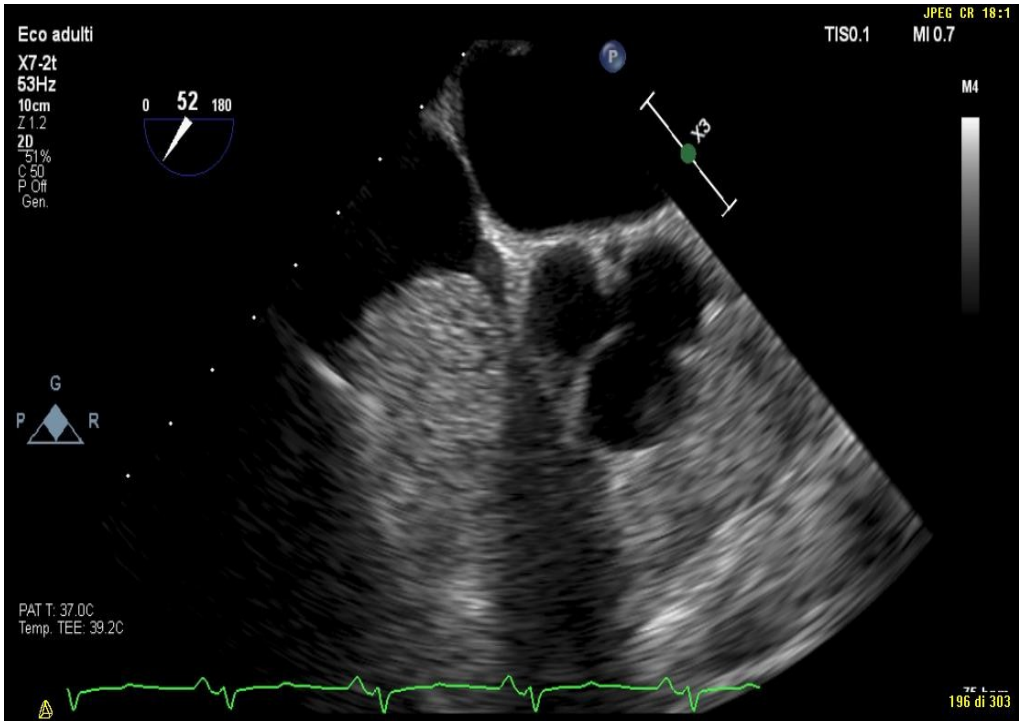

(c)

Figure 1. (a) Atrial septal defect (ASD) and patent foramen ovale (PFO) with right-to-left shunt; (b) Eustachian valve; (c) Eustachian valve after agitated saline solution injection. 
transthoracic echocardiography (one month and 12 months after surgery). She will receive double antiaggregation with aspirin and clopidogrel for three months, then only aspirin indefinitely if a significant shunt remains.

\section{Discussion}

The Eustachian valve rarely persists into adulthood but it needs to be carefully examined with transesophageal echocardiography for diagnosis and possible hemodynamic and clinic impact, especially for an increased incidence of cerebral embolism, including ischemic stroke.

Several evidences support the hypothesis that the association of Eustachian valve (especially the lenght of the EV) with PFO can represent a way faciliting systemic embolism. Our case shows how specific anatomical features, such as the persistence of Eustachian valve has increased the risk of embolic ischemic stroke, which need to be submitted to surgery especially for complex cardiac anatomy.

Considering that is known the association between migraine and cerebral embolism, the headache of the patient may be explained by this kind of relationship.

In addiction several studies have investigated the role of the persistence of Eustachian valve in adult life in the pathophysiology of endocarditis, especially Methicillin-Resistant Staphylococcus aureus endocarditis. In general, transesophageal echocardiography is the preferred method for visualization right-sided vegetations. In our case we didn't document endocarditis vegetations with echocardiography but the finding of $S$. aureus in blood cultures increases the suspect that there is a relationship between the bacteraemia and the Eustachian valve.

\section{Conclusions}

Our case report suggests the correlation between the persistence of Eustachian valve and PFO in adulthood and cryptogenic stroke, underscoring the importance to diagnose and submit it to endovascular surgery for the closure of the cardiac defect.

The known migraine of the patient may be interpreted as a warning symptom of cerebral embolism.

Finally, even if we haven't documented endocarditis vegetations with transesophageal echocardiography, the finding of $S$. aureus in blood cultures may not be independent of the patient's cardiac anatomy.

\section{Conflicts of Interest}

The authors declare no conflicts of interest regarding the publication of this paper.

\section{References}

[1] Sun, Y.P. and Homma S. (2016) Patent Foramen Ovale and Stroke. Circulation Journal, 80, 1665-1673. https://doi.org/10.1253/circj.CJ-16-0534 
[2] Mojadidi, M.K., Zaman, M.O., Elgendy, I.Y., Mahmoud, A.N., Patel, N.K., Agarwal, N., Tobis, J.M. and Meier, B. (2018) Cryptogenic Stroke and Patent Foramen Ovale. Journal of American College of Cardiology, 71, 1035-1043. https://doi.org/10.1016/j.jacc.2017.12.059

[3] Savino, K., Lilli, A., Pensi, P., Parisse, I. and Ambrosio, G. (2007) Valvola di Eustachio prominente simulante una massa atriale destra. Giornale Italiano di Cardiologia, 8, 43-46

[4] Vale, T.A., Newton, J.D., Orchard, E., Bhindi, R., Wilson, N. and Ormerod, O.J. (2011) Prominence of the Eustachian Valve in Paradoxical Embolism. European Journal of Echocardiography, 12, 33-36. https://doi.org/10.1093/ejechocard/jeq100

[5] Rigatelli, G., Dell'avvocata, F., Cardaioli, P., Giordan, M., Braggion, G., Aggio, S., Roncon, L. and Chinaglia, M. (2008) Migraine-Patent Foramen Ovale Connection: Role of Prominent Eustachian Valve and Large Chiari Network in Migrainous Patients. The American Journal of the Medical Sciences, 336, 458-461.

https://doi.org/10.1097/MAJ.0b013e31816e189d

[6] Fan, J., Le, P.T.N. and Jones, B.D. (2019) Eustachian Valve Endocarditis. Proceedings (Baylor University Medical Center), 32, 572-573.

https://doi.org/10.1080/08998280.2019.1646595

[7] W.L. Gore and Associates, Inc., Newark. 